\title{
Impact of glucose excursion and mean glucose concentration in oral glucose-tolerance test on oxidative stress among Japanese Americans
}

This article was published in the following Dove Press journal:

Diabetes, Metabolic Syndrome and Obesity:Targets and Therapy

2I November 2013

Number of times this article has been viewed

\section{Shuhei Nakanishi \\ Masayasu Yoneda \\ Shusaku Maeda}

Department of Molecular and Internal Medicine, Graduate

School of Biomedical and Health Sciences, Hiroshima University,

Hiroshima, Japan
Correspondence: Shuhei Nakanishi Department of Molecular and Internal Medicine, Graduate School of Biomedical and Health Sciences, Hiroshima University, I-2-3 Kasumi, Minami-ku, Hiroshima 734-855I, Japan

Tel +8I 822575196

Fax +8I 822557360

Email n-shuhei@umin.net
Aim: To evaluate the impact of glucose excursion (GE) and mean glucose concentration (MGC) on oxidative stress among persons with or without diabetes.

Materials and methods: We examined 775 Japanese Americans who had normal glucose tolerance (NGT), impaired glucose tolerance, or diabetes according to the $75 \mathrm{~g}$ oral glucosetolerance test, using fasting, 1-hour, and 2-hour glucose data. We calculated GE by subtracting the minimum from the maximum glucose level among three points and calculated MGC using these measurements. We investigated the relationship between GE or MGC and urinary isoprostane as a marker of oxidative stress.

Results: According to tertiles of GE or MGC, GE was associated with isoprostane levels among subjects with NGT as well as those with diabetes ( $P=0.004$ and 0.033 for trend, respectively). However, MGC was associated with isoprostane only among NGT subjects ( $P=0.001$ for trend). Association between GE and isoprostane was significant when adjustment was made for age, sex, smoking status, body mass index, C-reactive protein, glucose tolerance status, and homeostatic model assessment $(P=0.029)$, but the association with MGC was not significant.

Conclusion: Our results suggest the possibility that GE might result in oxidative stress, and the relationship is stronger than that for MGC.

Keywords: glucose excursion, glucose-tolerance test, oxidative stress

\section{Introduction}

Type 2 diabetes is characterized by a high incidence of cardiovascular disease (CVD). ${ }^{1}$ Diabetes patients exhibit two- to fourfold increased risk compared to subjects without diabetes, even when the analysis is adjusted for several cardiovascular risk factors. ${ }^{2}$ However, reduction of hyperglycemia expressed as glycated hemoglobin showed only a weak relation to risk of macrovascular complications compared with microvascular complications. ${ }^{3}$ On the other hand, recently there has been increasing evidence that postprandial hyperglycemia might play an important role in the development of atherosclerosis, ${ }^{4} \mathrm{CVD},{ }^{5}$ and diabetes mellitus (DM) complications. ${ }^{6}$ The DECODE (Diabetes Epidemiology: Collaborative analysis Of Diagnostic criteria in Europe) analysis reported that high blood glucose concentrations 2 hours after load in the glucose-tolerance test (GTT) are associated with increased risk of death, independently of fasting blood glucose. ${ }^{7}$ Also, the Funagata Diabetes Study ${ }^{8}$ and AusDiab (Australian Diabetes, Obesity, and Lifestyle Study) ${ }^{9}$ concluded that impaired glucose tolerance (IGT), but not impaired fasting glucose, is a risk factor for death from CVD. In addition, the LURIC (LUdwigshafen RIsk and Cardiovascular health) study reported that postchallenge hyperglycemia identified a high-risk group for CVD mortality 
undetected by fasting glucose and/or glycated hemoglobin. ${ }^{10}$ These findings suggest that temporary hyperglycemia, ie, high levels of glucose excursion (GE), is associated with the development of CVD.

Activation of oxidative stress is thought to be related to injury of various organs, with evidence increasingly indicating that oxidative stress may play an important role in DM vascular complications. ${ }^{11}$ Several in vitro studies have demonstrated increased expression of markers of oxidative stress in cells exposed to high GE. ${ }^{12,13}$ In addition, GE data that were obtained from continuous glucose monitoring carried out during the day indicated a more specific triggering effect on oxidative stress than chronic sustained hyperglycemia among type 2 DM patients. ${ }^{14}$ However, not only is the impact of GE or glucose elevation on oxidative stress unclear in subjects with or without diabetes, but how the effect differs with differing glucose-tolerance status is also unclear. Therefore, we compared GE and glucose elevation derived from a $75 \mathrm{~g}$ oral GTT with urine isoprostane, a well-recognized marker of oxidative stress, ${ }^{15,16}$ to study the impacts of GE by subtracting the minimum from the maximum glucose value among fasting, 1-hour, and 2-hour afterload of GTT, or mean glucose concentration (MGC) from all three glucose values, and different glucose-tolerance status on oxidative stress in Japanese Americans living in Hawaii and Los Angeles, who share a virtually identical genetic makeup with native Japanese currently living in Japan. In a previous study, we demonstrated that the prevalence as well as incidence of type $2 \mathrm{DM}$ and carotid intima-media thickness were significantly higher in Japanese Americans than in native Japanese, which suggests that the Westernization of lifestyle may promote the development of preclinical atherosclerosis in Japanese. ${ }^{17}$

\section{Materials and methods Experimental design}

This survey was part of a long-term epidemiological study initiated in 1970 of risk factors for DM and CVD, in which the subjects were limited to a population genetically identical to the Japanese population. The epidemiological study has previously been described in detail elsewhere. ${ }^{17,18}$ The subjects of our study were 775 Japanese Americans (302 men and 473 women) enrolled in a medical survey conducted in Hawaii in 2002 or Los Angeles in 2004, including persons who were under treatment for hyperlipidemia and/or hypertension. All subjects had glucose-tolerance status ascertained by GTT and were diagnosed according to the 1998 World Health Organization criteria. ${ }^{19}$ They had not been diagnosed with DM previously, and were free of infectious symptoms, autoimmune disease, and other acute conditions, as assessed by medical interview. Smoking status (current, past, or never-smoker) was assessed using standard interviewing procedures. All subjects underwent physical measurements and provided blood and urine samples after an overnight fast. Subjects with serum creatinine levels higher than $2 \mathrm{mg} / \mathrm{dL}$ were excluded from the study. The collected blood was centrifuged; serum, whole blood, and urine samples were immediately frozen and subsequently sent to Japan for analysis. Insulin was measured by double-antibody radioimmunoassay. Urinary isoprostane concentration was determined by enzyme immunoassay (Oxford Biomedical Research, Rochester Hills, MI, USA). ${ }^{20}$ Urine data were expressed as the ratio relative to urinary creatinine level measured in the same sample. C-reactive protein (CRP) levels were measured using a highly sensitive, latex-enhanced immunonephelometric assay. ${ }^{21}$ Insulin resistance was evaluated using the homeostasis model assessment (HOMA) for insulin resistance. ${ }^{22}$ This study was approved by the ethics committee of Hiroshima University and the Council of the Hiroshima Kenjin-Kai Association in Hawaii and Los Angeles.

\section{Statistical analysis}

Data are summarized as means and standard error. Because triglycerides, CRP, and isoprostane did not exhibit normal distributions, those data were analyzed after logarithmic transformation. As mentioned above, we calculated GE by subtracting the minimum from the maximum glucose value among fasting, 1-hour, and 2-hour afterload of GTT, and calculated MGC from all three glucose values. Continuous variables were first assessed using analysis of covariance, and if they were found to be significant, the Tukey-Kramer method was used to assess relationships among categories. Subjects were then divided into tertiles according to values of MGC or GE, calculated separately for each group undergoing 75 g GTT. Regarding MGC, we divided the normal glucose tolerance (NGT) group into categories comprised of less than $5.5,5.5-<6.5$, and $6.5 \mathrm{mmol} / \mathrm{L}$ or more; for the IGT group, categories of less than 7.8, 7.8-<8.6, and $8.6 \mathrm{mmol} / \mathrm{L}$ or more; and for the DM group, less than 10.7, $10.7-<13.0$, and $13.0 \mathrm{mmol} / \mathrm{L}$ or more. As for GE, we divided the NGT group into categories of less than 2.16, $2.16-<3.77$, and $3.77 \mathrm{mmol} / \mathrm{L}$ or more; for the IGT group, less than $4.72,4.72-<6.11$, and $6.11 \mathrm{mmol} / \mathrm{L}$ or more; and for the DM group, less than 7.77, 7.77-<8.88, and $8.88 \mathrm{mmol} / \mathrm{L}$ or more. We compared these concentrations with isoprostane levels in the three categories within each group. Numbers of subjects according to MGC tertile were 
185, 186, and 187 for the NGT group, 58, 52, and 55 for the IGT group, and 16, 19, and 17 for the DM group. Numbers of subjects according to GE tertile were 184, 191, and 183 for the NGT group, 54, 52, and 59 for the IGT group, and 19, 17, and 16 for the DM group. Multiple regression analyses were performed to investigate associations between urinary isoprostane as the outcome variable and either MGC or GE as the explanatory variable, after adjusting for age, sex, smoking status, body mass index (BMI), CRP, HOMA, and glucose-tolerance status (NGT, IGT, or DM). Age, CRP, BMI, and HOMA were used as continuous variables. Sex, smoking and glucose-tolerance status were used as categorical variables. SAS version 8.2 (SAS Institute, Cary, NC, USA) was used for all analyses.

\section{Results}

Comparisons among groups in terms of clinical characteristics of subjects after adjusting for age, sex, and smoking status are shown in Table 1. Systolic blood pressure, BMI, fasting glucose and insulin, 1-hour and 2-hour glucose and insulin, triglycerides, high-density lipid cholesterol, and CRP were all significantly higher in subjects with DM than in NGT subjects. Both MGC and GE were significantly

Table I Clinical characteristics of subjects

\begin{tabular}{|c|c|c|c|}
\hline & NGT & IGT & DM \\
\hline $\mathrm{N}$ (men/women) & $558(210 / 348)$ & $165(66 / 99)$ & $52(26 / 26)$ \\
\hline Age (years) & $63.1 \pm 0.6$ & $67.4 \pm 1.1^{\dagger}$ & $68.7 \pm 1.8^{\dagger}$ \\
\hline SBP $(\mathrm{mmHg})$ & $135 \pm 1$ & $138 \pm 1^{\dagger}$ & $140 \pm 2^{\dagger}$ \\
\hline $\mathrm{DBP}(\mathrm{mmHg})$ & $76 \pm 1$ & $77 \pm 1$ & $78 \pm 1$ \\
\hline BMI & $23.7 \pm 0.2$ & $25.0 \pm 0.3^{\dagger}$ & $25.6 \pm 0.5^{\dagger}$ \\
\hline Fasting glucose $(\mathrm{mmol} / \mathrm{L})$ & $4.89 \pm 0.04$ & $5.18 \pm 0.06^{\dagger}$ & $7.33 \pm 0.11^{1+. \neq}$ \\
\hline I-hour glucose (mmol/L) & $7.84 \pm 0.11$ & $10.67 \pm 0.18^{\dagger}$ & $15.05 \pm\left. 0.3\right|^{t . \neq}$ \\
\hline 2-hour glucose $(\mathrm{mmol} / \mathrm{L})$ & $5.5 I \pm 0.08$ & $8.84 \pm 0.12^{\dagger}$ & $14.58 \pm 0.22^{+, \neq}$ \\
\hline Fasting insulin (pmol/L) & $41.5 \pm 1.7$ & $51.9 \pm 2.8^{+}$ & $71.1 \pm 4.8^{\dagger, \neq}$ \\
\hline I-hour insulin (pmol/L) & $402.8 \pm 14.2$ & $473.7 \pm 23.0^{\dagger}$ & $351.6 \pm 39.9 \ddagger$ \\
\hline 2-hour insulin (pmol/L) & $262.8 \pm 12.5$ & $529.4 \pm 20.3^{\dagger}$ & $438.6 \pm 35.3^{t . \neq}$ \\
\hline $\mathrm{T}-\mathrm{CHO}(\mathrm{mmol} / \mathrm{L})$ & $5.58 \pm 0.05$ & $5.59 \pm 0.08$ & $5.65 \pm 0.14$ \\
\hline $\mathrm{TG}(\mathrm{mmol} / \mathrm{L})$ & $1.58 \pm 0.07$ & $1.85 \pm 0.11^{\dagger}$ & $2.46 \pm 0.19^{\dagger}$ \\
\hline HDL-C (mmol/L) & $1.48 \pm 0.02$ & $1.40 \pm 0.03$ & $1.32 \pm 0.05^{\dagger}$ \\
\hline $\mathrm{MGC}(\mathrm{mmol} / \mathrm{L})$ & $6.08 \pm 0.06$ & $8.23 \pm 0.10^{\dagger}$ & $12.32 \pm 0.18^{t . \neq}$ \\
\hline $\mathrm{GE}(\mathrm{mmol} / \mathrm{L})$ & $3.30 \pm 0.09$ & $5.65 \pm 0.14^{\dagger}$ & $8.5 \mathrm{I} \pm 0.24^{\dagger, f}$ \\
\hline CRP (mg/L) & $0.19 \pm 0.02$ & $0.20 \pm 0.03^{\dagger}$ & $0.24 \pm 0.05^{\dagger}$ \\
\hline $\begin{array}{l}\text { Urinary isoprostane } \\
(\mathrm{ng} / \mathrm{gCr})\end{array}$ & $0.59 \pm 0.05$ & $0.68 \pm 0.08$ & $1.00 \pm 0.13$ \\
\hline
\end{tabular}

Notes: ${ }^{\dagger} P<0.05$ toward NGT; $\ddagger P<0.05$ toward IGT by Tukey-Kramer method. Data are expressed as means \pm standard error. All data are adjusted for age, sex, and smoking status except age.

Abbreviations: NGT, normal glucose tolerance; IGT, impaired glucose tolerance; DM, diabetes mellitus; SBP, systolic blood pressure; DBP, diastolic blood pressure; BMI, body mass index; T-CHO, total cholesterol; TG, triglycerides; HDL-C, high density lipoprotein cholesterol; MGC, mean glucose concentration; GE, glucose excursion; CRP, C-reactive protein; $\mathrm{Cr}$, creatinine. higher in DM and in IGT subjects than in NGT subjects. Additionally, in IGT subjects, systolic blood pressure, BMI, fasting glucose and insulin, 1-hour glucose and insulin, 2-hour glucose and insulin, triglycerides, and CRP were significantly higher than in NGT subjects.

Urinary isoprostane levels with adjustment for age, sex, and smoking status after dividing subjects into tertiles of MGC levels in NGT, IGT, and DM subjects are shown in Figure 1. Among NGT subjects, urinary isoprostane levels with increasing MGC tertile were $0.52 \pm 0.06,0.54 \pm 0.06$, and $0.75 \pm 0.06 \mathrm{ng} / \mathrm{gCreatinine}(\mathrm{Cr})(P=0.0002$ for trend $)$, with the high category of MGC having significantly higher isoprostane levels than the low category $(P=0.002)$. Among IGT subjects, these levels were $0.59 \pm 0.16,0.62 \pm 0.16$, and $0.80 \pm 0.16 \mathrm{ng} / \mathrm{gCr}$ (trend not statistically significant). Among DM subjects, levels were 0.71 \pm 0.54 , 1.04 \pm 0.47 , and $1.03 \pm 0.53 \mathrm{ng} / \mathrm{gCr}$ (no trend). Regarding the GE tertiles, urinary isoprostane levels were $0.49 \pm 0.06,0.62 \pm 0.06$, and $0.68 \pm 0.06 \mathrm{ng} / \mathrm{gCr}$ among NGT subjects $(P=0.0016$ for trend), with the middle and high categories of GE having significantly higher isoprostane levels than the low category ( $P=0.045$ and 0.002 , respectively) (Figure 2 ). Among IGT subjects, these levels were $0.60 \pm 0.16,0.41 \pm 0.16$, and $0.97 \pm 0.15 \mathrm{ng} / \mathrm{gCr}$ (trend not statistically significant). Among DM subjects, the levels were $0.45 \pm 0.47,0.98 \pm 0.51$, and $1.41 \pm 0.49 \mathrm{ng} / \mathrm{gCr}(P=0.044$ for trend $)$, with the high categories of GE having significantly higher isoprostane levels than the low category $(P=0.045)$.

Using regression modeling to investigate associations between MGC or GE and urinary isoprostane (Tables 2 and 3, respectively), MGC was significantly associated with isoprostane $(P=0.010)$ without adjustment for other factors. After adjustment for age, sex, and smoking status, the association with isoprostane remained significant $(P=0.004)$. The statistically significant association remained with further adjustment for BMI, CRP, glucosetolerance status, or HOMA ( $P=0.012,0.014,0.047$, and 0.036 , respectively). However, the association disappeared $(P=0.127)$ after adjusting for all of the above factors. Isoprostane was significantly associated with GE $(P=0.0009)$, having a positive slope without adjustment for other factors. After adjustment for age, sex, and smoking status, the positive association remained significant $(P=0.0002)$. With further adjustment for BMI, CRP, glucose-tolerance status, and HOMA, the association remained significant ( $P=0.0007,0.0009,0.0016$, and 0.0017 , respectively). The association remained significant even after adjusting for all of the factors $(P=0.0049)$. 


\section{Tertile of MGC}
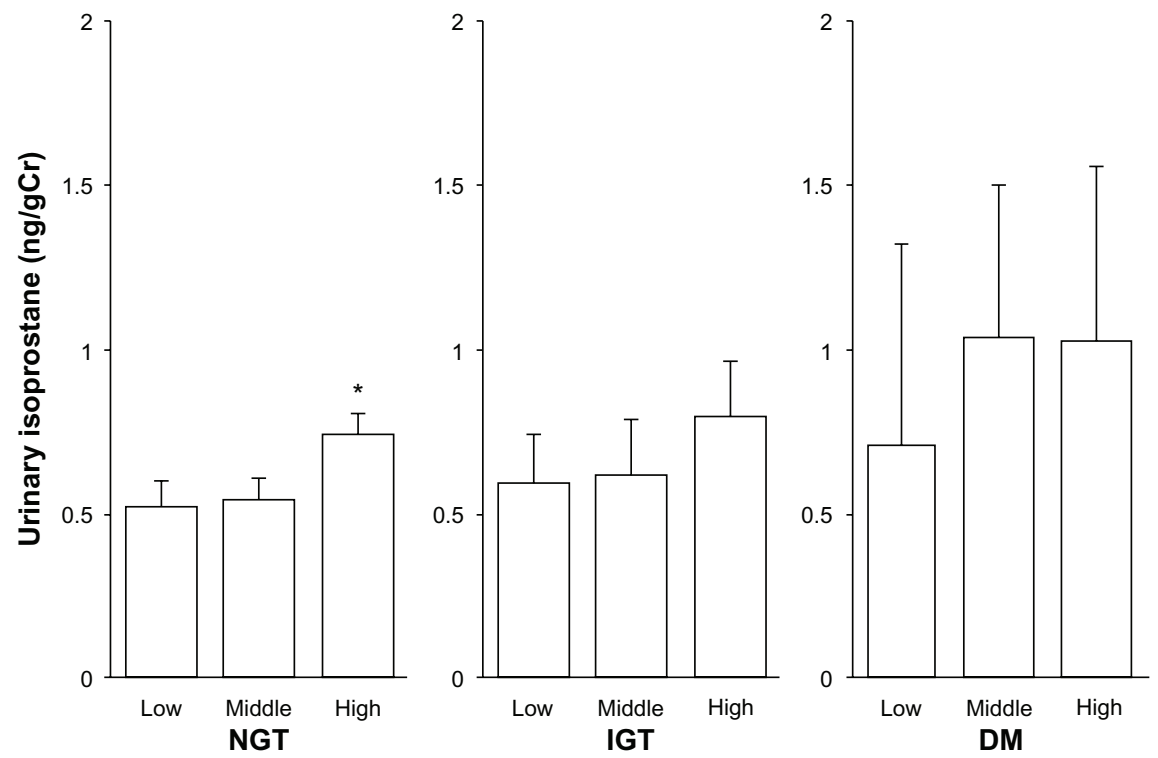

Figure I Relationship between urinary isoprostane level and mean glucose concentration (MGC).

Notes: Urinary isoprostane levels were grouped according to tertile of MGC. Data are expressed according to glucose-tolerance test diagnosis. Bars indicate standard error. Mean MGC levels among the normal glucose tolerance (NGT) subjects, from lowest to highest tertile, were 4.88 $\pm 0.49,6.02 \pm 0.29$, and 7.20 \pm 0.56 mmol/L, respectively. Among impaired glucose tolerance (IGT) subjects, these levels were $7.29 \pm 0.34,8.20 \pm 0.23$, and $9.20 \pm 0.46 \mathrm{mmol} / \mathrm{L}$, respectively. Among the subjects with diabetes mellitus (DM), these levels were $9.85 \pm 0.83, \mathrm{II} .75 \pm 0.68$, and I5.32 $\pm 3.5 \mathrm{I} \mathrm{mmol} / \mathrm{L}$, respectively. Numbers of subjects according to MGC tertile were I85, I86, and I87 for the NGT group, 58,52 , and 55 for the IGT group, and 16, 19, and 17 for the DM group. Trend analysis revealed $P=0.0002, P=0.45$ I, and $P=0.743$ among NGT, IGT, and subjects with diabetes, respectively. $* P<0.05$ compared with the lowest tertile by the Tukey-Kramer method.

Abbreviation: $\mathrm{Cr}$, creatinine.

\section{Tertile of GE}
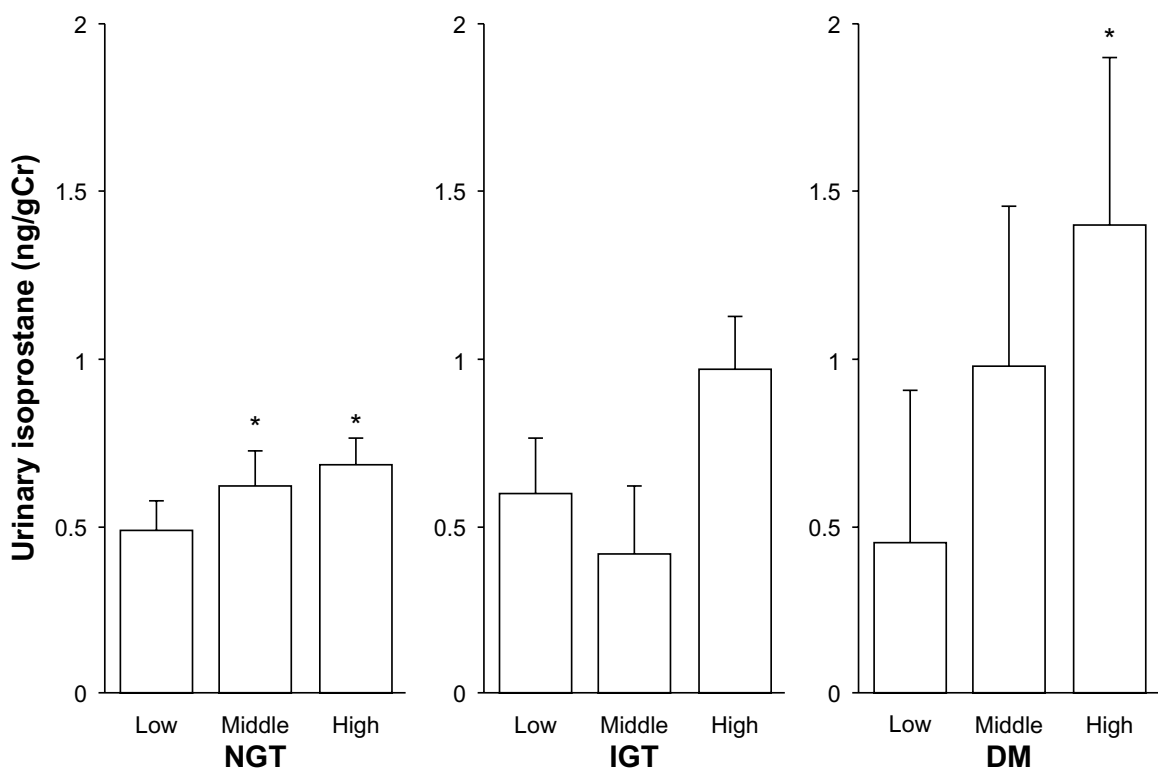

Figure 2 Relationship between urinary isoprostane level and glucose excursion (GE).

Notes: Urinary isoprostane levels were grouped according to tertile of GE. Data are expressed according to glucose-tolerance test diagnosis. Bars indicate standard error. Mean GE levels among normal glucose tolerance (NGT) subjects, from lowest to highest tertile, were $1.32 \pm 0.57,3.01 \pm 0.46$, and $5.2 I \pm I$. I0 mmol/L, respectively. Among the impaired glucose tolerance (IGT) subjects, these levels were $3.89 \pm 0.65,5.46 \pm 0.39$, and $7.17 \pm 0.8 \mathrm{I} \mathrm{mmol} / \mathrm{L}$, respectively. Among the subjects with diabetes mellitus (DM), these levels were $6.8 \mathrm{I} \pm 0.9 \mathrm{I}, 8.38 \pm 0.33$, and I0.54 $\pm \mathrm{I} .5 \mathrm{I} \mathrm{mmol} / \mathrm{L}$, respectively. Numbers of subjects according to GE tertile were I84, I9I, and I83 for the NGT group, 54 , 52 , and 59 for the IGT group, and 19, I7, and 16 for the DM group. Trend analysis revealed $P=0.002, P=0.350$, and $P=0.044$ among NGT, IGT, and subjects with diabetes, respectively. $* P<0.05$ compared with the lowest tertile by the Tukey-Kramer method.

Abbreviation: $\mathrm{Cr}$, creatinine. 
Table 2 The relationship of MGC by regression analysis with urinary isoprostane as the dependent variable

\begin{tabular}{|c|c|c|c|}
\hline Adjustment & $\beta$ & SE & $\begin{array}{l}P \\
\text {-value }\end{array}$ \\
\hline MGC only & 0.043 & 0.016 & 0.010 \\
\hline Adjusted for age, sex, and smoking status & 0.048 & 0.017 & 0.004 \\
\hline $\begin{array}{l}\text { Adjusted for age. sex, smoking status, } \\
\text { and BMI }\end{array}$ & 0.043 & 0.017 & 0.012 \\
\hline $\begin{array}{l}\text { Adjusted for age, sex, smoking status, } \\
\text { and CRP }\end{array}$ & 0.042 & 0.017 & 0.014 \\
\hline $\begin{array}{l}\text { Adjusted for age, sex, smoking status, } \\
\text { and glucose tolerance status }\end{array}$ & 0.055 & 0.028 & 0.047 \\
\hline $\begin{array}{l}\text { Adjusted for age, sex, smoking status, } \\
\text { and HOMA }\end{array}$ & 0.039 & 0.019 & 0.036 \\
\hline $\begin{array}{l}\text { Adjusted for age, sex, smoking status, BMI, } \\
\text { CRP, HOMA, and glucose tolerance status }\end{array}$ & 0.045 & 0.029 & 0.127 \\
\hline
\end{tabular}

Abbreviations: SE, standard error; MGC, mean glucose concentration; BMI, body mass index; CRP, C-reactive protein; HOMA, homeostatic model assessment for insulin resistance.

\section{Discussion}

The results of the present study show that GE as well as MGC (both calculated using GTT data) were related to the potential production of oxidative stress, as indicated by urinary isoprostane, whereas the relationship for GE was stronger than that for MGC. The results suggest the possibility that excursions in glucose level during the GTT might be effective for inferring a high risk of CVD not only among subjects with type $2 \mathrm{DM}$ but even among subjects who do not have glucose intolerance.

The relationship between oxidative stress and insulin resistance, leading to type $2 \mathrm{DM}$, and endothelial dysfunction, leading to CVD, has received considerable attention. Recent studies demonstrate that hyperglycemia induces an overproduction of oxidative stress by the mitochondrial electron-transport chain. ${ }^{23,24} \mathrm{An}$ increase of plasma malon-

Table 3 The relationship of GE by regression analysis with urinary isoprostane as the dependent variable

\begin{tabular}{|c|c|c|c|}
\hline Adjustment & $\beta$ & SE & $\begin{array}{l}P \\
\text {-value } \\
\end{array}$ \\
\hline GE only & 0.050 & 0.015 & 0.0009 \\
\hline Adjusted for age, sex, and smoking status & 0.057 & 0.015 & 0.0002 \\
\hline $\begin{array}{l}\text { Adjusted for age, sex, smoking status, } \\
\text { and BMI }\end{array}$ & 0.053 & 0.016 & 0.0007 \\
\hline $\begin{array}{l}\text { Adjusted for age, sex, smoking status, } \\
\text { and CRP }\end{array}$ & 0.052 & 0.016 & 0.0009 \\
\hline $\begin{array}{l}\text { Adjusted for age, sex, smoking status, } \\
\text { and glucose tolerance status }\end{array}$ & 0.065 & 0.021 & 0.0016 \\
\hline $\begin{array}{l}\text { Adjusted for age, sex, smoking status, } \\
\text { and HOMA }\end{array}$ & 0.051 & 0.016 & 0.0017 \\
\hline $\begin{array}{l}\text { Adjusted for age, sex, smoking status, BMI, } \\
\text { CRP, HOMA, and glucose tolerance status }\end{array}$ & 0.059 & 0.021 & 0.0049 \\
\hline
\end{tabular}

Abbreviations: SE, standard error; GE, glucose excursion; BMI, body mass index CRP, C-reactive protein; HOMA, homeostatic model assessment for insulin resistance. dialdehyde and a decrease of circulating antioxidants were found both in DM and normal subjects during the GTT. ${ }^{25}$ In addition, Kawano et al reported that endothelium-dependent vasodilation in NGT subjects decreased through increased oxidative stress 1 hour after glucose loading. ${ }^{26}$ Thus, considering our results, the possibility is indicated that NGT subjects whose GE was high in the GTT might also have increased levels of oxidative stress, because subjects with high GE can be assumed to also have high postprandial GE. These findings are consistent with evidence showing that postprandial hyperglycemia may play an important role in the development and progression of atherosclerosis, even among NGT subjects. ${ }^{26}$ However, the relationship between GE and oxidative stress is still conflicting, even among subjects with type 2 $\mathrm{DM},{ }^{14,27}$ as well as among healthy volunteers. ${ }^{28}$ Concerning two studies, ${ }^{14,27}$ subjects with type $2 \mathrm{DM}$ were recruited from an outpatient clinic. In addition, healthy volunteers in another study ${ }^{28}$ were not ascertained using GTT. In contrast, subjects in our study had not been diagnosed with DM previously, and had been determined by their glucose tolerance status, by GTT. To understand the differences, further study on this matter is required.

MGC was correlated with urinary isoprostane only among the NGT subjects in our study. A previous study that examined subjects with DM over a 24-hour period also demonstrated a significant relationship between activation of oxidative stress and acute glucose swings, but did not examine any parameters of long-term glucose exposure. ${ }^{14}$ One reason for the apparent lack of association among IGT and DM subjects is that compared to NGT subjects, continuous hyperglycemic states are thought to elevate oxidative stress for a number of reasons, ${ }^{24}$ leading to elevated isoprostane level. In addition, during chronic exposure to high levels of glucose, some adaptive mechanisms might work against certain toxic glucose effects. ${ }^{29}$ As a result, the impact of MGC on oxidative stress might have been somewhat attenuated. Further study of this issue is also required.

We found that the oxidative stress marker was strongly correlated with GE among the NGT subjects. Several studies that support our data have assessed the comparison between the impact of GE and that of continuous hyperglycemia on various factors related to oxidative stress. Recent studies using rats with or without DM demonstrated that postprandial GE evokes greater levels of monocyte adhesion to endothelial cells of the thoracic aorta than that induced by stable hyperglycemia. ${ }^{30,31}$ Furthermore, other studies have demonstrated that oxidative stress production is more pronounced and apoptosis levels are increased under exposure 
to intermittently high glucose compared with exposure to constantly high glucose levels in human umbilical vein endothelial cells. ${ }^{12,29}$ On the other hand, it has been demonstrated that oxidative stress could be diminished by reducing postprandial GE, defined as the change in glucose concentration from before to after meal intake. ${ }^{32}$ Taking these findings into account, when we examine GTT, we must consider not only glucose data for diagnosing DM or glucose intolerance but also excursion in glucose levels for understanding the latent risk of CVD through high oxidative stress.

Although the mechanism underlying different roles of MGC and GE in oxidative stress remains unknown at present, possible explanations include the following. The effect of MGC on oxidative stress tended to be confounded by glucose intolerance and insulin resistance in our study, because the significance of the relationship between urinary isoprostane and MGC was markedly attenuated after further adjustment for glucose-tolerance status or HOMA (in addition to age, sex, and smoking status; Table 2). In fact, the significance of these relationships disappeared after adjustment for age, sex, HOMA, and glucose-tolerance status $(P=0.112$, data not shown). In addition, both high glucose level and insulin resistance are thought to be related to oxidative stress. ${ }^{12,24,33}$ Therefore, MGC may be associated with oxidative stress through insulin resistance or glucose metabolism. On the contrary, the effect of GE on oxidative stress also tended to be confounded by insulin resistance, as shown in Table 3 . However, the attenuation was small compared to that of MGC. GE may be associated with oxidative stress through protein kinase $\mathrm{C}$-dependent activation of nicotinamide adenine dinucleotide phosphate oxidase, ${ }^{12}$ which was greater in the fluctuating than in the stable high-glucose condition in endothelial cells, or activation of the ras-mitogen-activated protein kinase pathway and nuclear translocation of nuclear factor- $\kappa \mathrm{B},{ }^{13}$ independent of insulin resistance indicated by HOMA.

This study has several limitations. First, medications were not fully considered. However, there were no differences in GE, MGC, or urinary isoprostane levels between subjects who were not under treatment for hyperlipidemia or hypertension and subjects who were being treated (data not shown). Second, renal function might influence urinary isoprostane levels, leading to the possibility of a misunderstanding of the relationship between GE or MGC and oxidative stress. However, patients with severe renal dysfunction were excluded: all subjects' serum creatinine levels in this study were under $2 \mathrm{mg} / \mathrm{dL}$. Third, the study was cross-sectional; we might have overestimated the relationships between
GE or MGC and oxidative stress because we were unable to distinguish cause from effect. To allow more general conclusions, more detailed studies employing alternative designs are needed.

In summary, GE calculated from GTT data was related to the potential induction of oxidative stress not only among subjects with diabetes but also among NGT subjects, whereas MGC was related only among NGT subjects. The results suggest that GTT might be a useful tool for use in detecting a high risk of CVD as well as defining glucose intolerance.

\section{Acknowledgments}

The authors wish to thank the members of the Hiroshima Kenjin-Kai of Hawaii and Southern California for their participation. We are indebted to the late Dr Seiryo Takashina, former president of Hiroshima General Hospital, and Dr Kazufumi Ishida and Dr Hirofumi Usui, Hiroshima General Hospital, for performing sample assays.

\section{Disclosure}

The authors report no conflicts of interest in this work.

\section{References}

1. Kannel WB, McGee DL. Diabetes and cardiovascular disease. The Framingham study. JAMA. 1979;241(19):2035-2038.

2. Stamler J, Vaccaro O, Neaton JD, Wentworth D. Diabetes, other risk factors, and 12-yr cardiovascular mortality for men screened in the Multiple Risk Factor Intervention Trial. Diabetes Care. 1993;16(2):434-444.

3. Stratton IM, Adler AI, Neil HA, et al. Association of glycaemia with macrovascular and microvascular complications of type 2 diabetes (UKPDS 35): prospective observational study. BMJ. 2000;321(7258): 405-412.

4. Bonora E, Muggeo M. Postprandial blood glucose as a risk factor for cardiovascular disease in type II diabetes: the epidemiological evidence. Diabetologia. 2001;44(12):2107-2114.

5. Sourij H, Saely CH, Schmid F, et al. Post-challenge hyperglycaemia is strongly associated with future macrovascular events and total mortality in angiographied coronary patients. Eur Heart $J$. 2010;31(13):1583-1590.

6. Ceriello A. Postprandial hyperglycemia and diabetes complications: is it time to treat? Diabetes. 2005;54(1):1-7.

7. [No authors listed]. Glucose tolerance and mortality: comparison of WHO and American Diabetes Association diagnostic criteria. The DECODE study group. European Diabetes Epidemiology Group. Diabetes Epidemiology: Collaborative analysis Of Diagnostic criteria in Europe. Lancet. 1999;354(9179):617-621.

8. Tominaga M, Eguchi H, Manaka H, Igarashi K, Kato T, Sekikawa A. Impaired glucose tolerance is a risk factor for cardiovascular disease, but not impaired fasting glucose. The Funagata Diabetes Study. Diabetes Care. 1999;22(6):920-924.

9. Barr EL, Zimmet PZ, Welborn TA, et al. Risk of cardiovascular and all-cause mortality in individuals with diabetes mellitus, impaired fasting glucose, and impaired glucose tolerance: the Australian Diabetes, Obesity, and Lifestyle Study (AusDiab). Circulation. 2007;116(2):151-157.

10. Silbernagel G, Sourij H, Grammer TB, et al. Isolated post-challenge hyperglycaemia predicts increased cardiovascular mortality. Atherosclerosis. 2012;225(1):194-199. 
11. Giugliano D, Ceriello A, Paolisso G. Oxidative stress and diabetic vascular complications. Diabetes Care. 1996;19(3):257-267.

12. Quagliaro L, Piconi L, Assaloni R, Martinelli L, Motz E, Ceriello A. Intermittent high glucose enhances apoptosis related to oxidative stress in human umbilical vein endothelial cells: the role of protein kinase $\mathrm{C}$ and NAD(P)H-oxidase activation. Diabetes. 2003;52(11):2795-2804.

13. Schiekofer S, Andrassy M, Chen J, et al. Acute hyperglycemia causes intracellular formation of CML and activation of ras, p42/44 MAPK, and nuclear factor $\mathrm{KB}$ in PBMCs. Diabetes. 2003;52(3):621-633.

14. Monnier L, Mas E, Ginet C, et al. Activation of oxidative stress by acute glucose fluctuations compared with sustained chronic hyperglycemia in patients with type 2 diabetes. JAMA. 2006;295(14):1681-1687.

15. Morrow JD, Hill KE, Burk RF, Nammour TM, Badr KF, Roberts LJ. A series of prostaglandin F2-like compounds are produced in vivo in humans by a non-cyclooxygenase, free radical-catalyzed mechanism. Proc Natl Acad Sci U SA. 1990;87(23):9383-9387.

16. Roberts LJ, Morrow JD. Measurement of F(2)-isoprostanes as an index of oxidative stress in vivo. Free Radic Biol Med. 2000;28(4):505-513.

17. Nakanishi S, Okubo M, Yoneda M, Jitsuiki K, Yamane K, Kohno N. A comparison between Japanese-Americans living in Hawaii and Los Angeles and native Japanese: the impact of lifestyle westernization on diabetes mellitus. Biomed Pharmacother. 2004;58(10):571-577.

18. Egusa G, Murakami F, Ito C, et al. Westernized food habits and concentrations of serum lipids in the Japanese. Atherosclerosis. 1993; 100(2):249-255.

19. Alberti KG, Zimmet PZ. Definition, diagnosis and classification of diabetes mellitus and its complications. Part 1: diagnosis and classification of diabetes mellitus provisional report of a WHO consultation. Diabet Med. 1998;15(7):539-553.

20. Keaney JF Jr, Larson MG, Vasan RS, et al. Obesity and systemic oxidative stress: clinical correlates of oxidative stress in the Framingham Study. Arterioscler Thromb Vasc Biol. 2003;23(3):434-439.

21. Nakanishi S, Yamane K, Kamei N, Okubo M, Kohno N. Elevated C-reactive protein is a risk factor for the development of type 2 diabetes in Japanese Americans. Diabetes Care. 2003;26(10):2754-2757.

22. Matthews DR, Hosker JP, Rundenski AS, Naylor BA, Treacher DF, Turner RC. Homeostasis model assessment: insulin resistance and $\beta$-cell function from fasting plasma glucose and insulin concentrations in man. Diabetologia. 1985;28(7):412-419.
23. Brownlee M. Biochemistry and molecular cell biology of diabetic complications. Nature. 2001;414(6865):813-820.

24. Nishikawa T, Edelstein D, Du XL, et al. Normalizing mitochondrial superoxide production blocks three pathways of hyperglycaemic damage. Nature. 2000;404(6779):787-790.

25. Ceriello A, Bortolotti N, Crescentini A, et al. Antioxidant defences are reduced during the oral glucose tolerance test in normal and noninsulin-dependent diabetic subjects. Eur J Clin Invest. 1998;28(4): 329-333.

26. Kawano H, Motoyama T, Hirashima O, et al. Hyperglycemia rapidly suppresses flow-mediated endothelium-dependent vasodilation of brachial artery. J Am Coll Cardiol. 1999;34(1):146-154.

27. Siegelaar SE, Barwari T, Kulik W, Hoekstra JB, DeVries JH. No relevant relationship between glucose variability and oxidative stress in well-regulated type 2 diabetes patients. J Diabetes Sci Technol. 2011;5(1):86-92.

28. Wakil A, Smith KA, Atkin SL, Kilpatrick ES. Short-term glucose variability in healthy volunteers is not associated with raised oxidative stress markers. Diabetes Obes Metab. 2012;14(11):1047-1049.

29. Risso A, Mercuri F, Quagliaro L, Damante G, Ceriello A. Intermittent high glucose enhances apoptosis in human umbilical vein endothelial cells in culture. Am J Physiol Endocrinol Metab. 2001;281(5): E924-E930.

30. Otsuka A, Azuma K, Iesaki T, et al. Temporary hyperglycaemia provokes monocyte adhesion to endothelial cells in rat thoracic aorta. Diabetologia. 2005;48(12):2667-2674.

31. Azuma K, Kawamori R, Toyofuku Y, et al. Repetitive fluctuations in blood glucose enhance monocyte adhesion to the endothelium of rat thoracic aorta. Arterioscler Thromb Vasc Biol. 2006;26(10): 2275-2280.

32. Ceriello A, Quagliaro L, Catone B, et al. Role of hyperglycemia in nitrotyrosine postprandial generation. Diabetes Care. 2002;25(8): 1439-1443.

33. Houstis N, Rosen ED, Lander ES. Reactive oxygen species have a causal role in multiple forms of insulin resistance. Nature. 2006; 440(7086):944-948.

\section{Publish your work in this journal}

Diabetes, Metabolic Syndrome and Obesity: Targets and Therapy is an international, peer-reviewed open-access journal committed to the rapid publication of the latest laboratory and clinical findings in the fields of diabetes, metabolic syndrome and obesity research. Original research, review, case reports, hypothesis formation, expert opinion and commentaries are all considered for publication. The manuscript management system is completely online and includes a very quick and fair peer-review system, which is all easy to use. Visit http://www.dovepress.com/testimonials.php to read real quotes from published authors. 